\title{
Experimental studies for the personalized application of boron neutron capture therapy to the treatment of cutaneous melanoma
}

\author{
Marina Carpano ${ }^{1}$, Gustavo Santa Cruz ${ }^{2}$, Carla Rodriguez ${ }^{1}$, Susana Nievas ${ }^{2}$, Maria Silvina Olivera $^{2}$, \\ Marina Perona ${ }^{1,3}$, Esteban Boggio ${ }^{3}$, Juan Longhino ${ }^{3}$, Mario Pisarev ${ }^{1,4}$, Guillermo Juvenal ${ }^{1,4}$, \\ Maria Alejandra Dagrosa ${ }^{1,4}$
}

${ }^{1}$ Radiobiology Department (CAC), National Atomic Energy Commission (CNEA), San Martin, Provincia de Buenos Aires, Argentina; ${ }^{2}$ BNCT Coordination Department (CAC), National Atomic Energy Commission (CNEA), San Martin, Provincia de Buenos Aires, Argentina; ${ }^{3}$ RA- ${ }^{6}$ (CAB), National Atomic Energy Commission (CNEA), Bariloche, Provincia de Rio Negro, Argentina; ${ }^{4}$ National Council of Scientific and Technical Research (CONICET), Ciudad Autónoma de Buenos Aires, Argentina

Contributions: (I) Conception and design: M Carpano, G Santa Cruz, J Longhino, MA Dagrosa; (II) Administrative support: G Santa Cruz, M Perona, G Juvenal, MA Dagrosa; (III) Provision of study materials or patients: M Carpano, G Santa Cruz, C Rodriguez, S Nievas, MS Olivera, M Perona; (IV) Collection and assembly of data: M Carpano, G Santa Cruz, C Rodriguez, S Nievas, MS Olivera, M Perona, E Boggio, J Longhino, MA Dagrosa; (V) Data analysis and interpretation: M Carpano, G Santa Cruz, E Boggio, J Longhino, MA Dagrosa; (VI) Manuscript writing: All authors; (VII) Final approval of manuscript: All authors.

Correspondence to: Maria Alejandra Dagrosa, PhD. Radiobiology Department (CAC), National Atomic Energy Commission (CNEA), Avenida General Paz 1499, (B1650KNA) San Martín, Provincia Buenos Aires, Argentina. Email: alejandradagrosa@gmail.com.

Background: Boron neutron capture therapy (BNCT) is a binary modality based on the nuclear reaction ${ }^{10} \mathrm{~B}(\mathrm{n}, \alpha)$ lithium-7 ( $\left.{ }^{7} \mathrm{Li}\right)$ that has been used to treat a variety of tumors, among these, cutaneous melanoma $(\mathrm{CM})$. In previous boron biodistribution studies in agreement with the personalized oncology, we have demonstrated that boronophenylalanine (BPA) uptake can be correlated with the tumoral temperature and viability. The main aim of these studies was to evaluate the relationship between tumoral temperature and the response to the complete BNCT.

Methods: Nude mice were implanted with human melanoma cells (Mel J) and divided into different groups (Control, NCT, BNCT I and BNCT II) and irradiated with the thermal neutron beam from RA-6 (4.96x $10 \% \mathrm{~cm}^{2} / \mathrm{sec}$ ) during 37 and 55 minutes respectively. Tumor and body temperatures were measured by Static Infrared Imaging (SIRI), and it was performed the following up of the animals.

Results: Tumor growth showed a complete growth inhibition during the first 20 days after treatment in both BNCT groups (BNCT I and BNCT II vs. Control $\mathrm{P}<0.001)$. Considering the analogy between the Fourier's Law of Heat Conduction and the Ohm's law of Electrical Conduction, the quantity (T_tum-T_ inf)/(T_body-T_tum) was analyzed as a function of Vf/ $/ \mathrm{Vi}$ (Final volume/Initial volume) ratio. A tendency to higher values of the temperature's ratios, was observed with respect to the degree of tumor control (BNCT I with a $\mathrm{R}^{2}$ of 0.3527 , BNCTII with a $\mathrm{R}^{2}$ of 0.3327 ) in agreement with previous boron biodistribution studies. The histology and immunohistochemical studies showed larger areas of necrosis and picnotic regions and a significant decrease of the Ki-67 antibody labeling in the BNCT II group evidencing important tumor damage.

Conclusions: tumoral characteristics, especially the temperature, could be used to plan a personalized treatment for each patient. As values of correlation between temperature and tumoral response showed to be weak, we considered to explore a new model of three dimension for heat transport process.

Keywords: Boron neutron capture therapy (BNCT); melanoma; static infrared imaging (SIRI); personalized oncology

Received: 30 November 2020; Accepted: 01 April 2021; Published: 30 June 2021.

doi: 10.21037/tro-20-61

View this article at: http://dx.doi.org/10.21037/tro-20-61 


\section{Introduction}

Boron neutron capture therapy (BNCT) is a unique type of radiation therapy that has been used to treat cancer, among them high grade gliomas and melanoma. The basis of BNCT is the neutron capture reaction that results when boron-10, a stable isotope, is irradiated with lowenergy thermal neutrons or also, higher-energy epithermal neutrons. In turn, these neutrons lose energy and rapidly become thermalized. This capture reaction produces high linear energy transfer (LET) alpha particles $\left({ }^{4} \mathrm{He}\right)$ and recoiling lithium-7 $\left({ }^{7} \mathrm{Li}\right)$ nuclei. The destructive effects of the particles are limited to boron containing cells since they have very short ranges in tissues $(5-9 \mu \mathrm{m})$. The average LET is high $\left({ }^{7} \mathrm{Li}, 162 \mathrm{keV} / \mu \mathrm{m} ;{ }^{4} \mathrm{He}, 196 \mathrm{keV} / \mu \mathrm{m}\right)$, and this results in densely ionizing radiation restricted to the path length of each particle. For BNCT to be successful, a sufficient amount of ${ }^{10} \mathrm{~B}$ must be selectively delivered to the tumor $\left(\sim 20-50 \mu \mathrm{g} / \mathrm{g}\right.$ or $\sim 10^{9}$ atoms/cell) (1).

In 1972, experimental studies on BNCT for cutaneous melanoma (CM) were initiated by Mishima at Kobe University in Japan (2). In 1987, after 15 years of basic research, his team initiated the first clinical use of BNCT to treat a patient with a metastatic melanoma to the skin of the left occipital region of the scalp, using paraboronophenylalanine (BPA) as the boron delivery agent (3). Later several other countries including Argentina, began clinical trials to treat patients with either cutaneous or metastatic melanoma $(4,5)$.

Over the past two decades, an increase in the incidence of $\mathrm{CM}$ has been reported. The survival rates of melanoma, like any type of cancer, worsen with advancing stage. CM progress from the primary site to the in-transit locations, regional or sentinel lymph nodes and beyond to the distant sites. Therefore, early diagnosis and surgical treatment before it spreads is the most effective treatment. Conversely, in advanced stages the effectiveness of the therapies is poor (6). Radiotherapy is a fundamental part of multimodal procedures for the treatment of cancer that are based on the potential capacity to produce tumor cell death and to suppress clonogenic survival. The positive correlation between the improvement of local control and the increase in the overall survival has been documented by different clinical trials. Despite all the achieved improvements, the efficacy of the radiotherapy is still limited by different biological, technological, and clinical constraints. BNCT has shown the possible overcome of some of these limitations, for example the intrinsic radiation resistance and the true pathoanatomical borders of tumors or areas at risk that are often difficult to reach or identify. However, the heterogeneity of each tumor and the response of the normal tissue against both radiotherapeutic modalities is still extensive (7). At the present time, Personalized Cancer Therapy based on the specific clinical, histopathological and anatomical imaging biomarkers could be a new option in cancer treatment planning (8).

In order to provide non-invasive tools for the follow up of melanoma patients in the Argentina BNCT clinical trial for malignant melanoma, dynamic infrared thermography was included as part of the procedure, before and after treatment (9). In the context of preclinical and experimental research protocols currently in progress at our institution, Static Infrared Imaging (SIRI) has been employed using a specially modified infrared camera that was adapted to calculate the body infrared radiance of small animals before BNCT irradiations. SIRI is a non-invasive and non-contact approach that allows the measurement and visualization of infrared radiation. Thermal imaging systems has been utilized in medicine for more than 50 years in different clinical settings (10). We have previously performed a study of mice body, tumor and surrounding skin temperatures. A direct correlation between tumor temperature, percentage of viable cells and BPA uptake was observed. We have found, for the first time, a significant tumor temperature $v s$. tumor boron concentration correlation, which followed an empirical rational dependence, with a good correlation coefficient $\left(\mathrm{R}^{2}=0.7\right)(11)$.

In this paper, the main goal was to evaluate the relationship between the tumor temperature and the response to the application of BNCT. These results will allow to demonstrate if this parameter, measured before irradiation, could be used as predictive indicators of boron tumor uptake, in order to adjust the neutron fluence to optimize BNCT for each individual patient.

We present the following article in accordance with the ARRIVE reporting checklist (available at http://dx.doi. org/10.21037/tro-20-61).

\section{Methods}

\section{Cell line}

The human MEL-J melanoma cell line was kindly supplied by Dr. José Mordoh, Instituto de Investigaciones Bioquímicas Leloir (12). Cells were incubated in a $5 \%$ $\mathrm{CO}_{2}$ humidified incubator and maintained in RPMI1640 
culture medium supplemented with $10 \%$ fetal bovine serum (FBS) and $1 \%$ antibiotics (streptomycin $(100 \mathrm{mg} / \mathrm{mL}$ ) and penicillin $(100 \mathrm{IU} / \mathrm{mL})$. The study was conducted in accordance with the Declaration of Helsinki (as revised in 2013).

\section{Animal model}

Sixty NIH nude male mice (aged 6-8 weeks) were inoculated subcutaneously (sc) in the right flank with a suspension of $3 \times 10^{6}$ of Mel-J cells. Animals were housed in covered cages and kept under aseptic conditions (13).

Experiments were performed under a project license (Protocol N3/2015 "Preclinical studies to optimize the application of BNCT for the treatments of melanoma and undifferentiated thyroid cancer") granted by the Argentine National Atomic Energy Commission Animal Care and Use Committee (CICUAL-CNEA) in compliance with the National Institute of Health (NIH USA) guide for the care and use of Laboratory animals.

\section{Experimental design}

All the mice bearing tumors with a tumor volume between 25 and $75 \mathrm{~mm}^{3}$ were individualized with an earmark and divided into the following 4 groups $(\mathrm{n}=9-12)$ :

(I) Control: without irradiation and BPA.

(II) NCT: irradiated with neutron beam (during $37 \pm$ $0.5 \mathrm{~min})$.

(III) BNCT I: irradiated with the neutron beam (during $37 \pm 0.5 \mathrm{~min})$ plus BPA $(350 \mathrm{mg} / \mathrm{kg}$ b.w).

(IV) BNCT II: irradiated with the neutron beam (during $55 \pm 0.5 \mathrm{~min})$ plus BPA $(350 \mathrm{mg} / \mathrm{kg}$ b.w).

Infrared thermography was used to measure body and tumor temperature of each mouse. Next, animals were transported by airplane to the Bariloche Atomic Center $(\mathrm{CAB})$ for the irradiation in the therapeutic BNCT neutron facility of the RA6 Reactor (In-air thermal neutron flux: $4.96 \times 10^{8} \mathrm{n} / \mathrm{cm}^{2} / \mathrm{sec}$ ).

The $30 \mathrm{mg}$ of BPA per milliliter $(0.14 \mathrm{M})$ solution of L-p BPA (Gliconix Corp; Durham, US) was aseptically prepared at a concentration of $30 \mathrm{mg}{ }^{10} \mathrm{BPA}$ per milliliter $(0.14 \mathrm{M})$ (14) and administered to the animals via intraperitoneal (ip) 60 minutes before the irradiation. Before the application of the treatments, the mice were anesthetized subcutaneously (sc) with diazepam $(5 \mathrm{mg} / \mathrm{kg}$ b.w) and 20 minutes after with ketamine (200 mg/kg b.w).

\section{Characteristics of the irradiation}

Animals were divided in groups of 8 individuals and irradiation was done on a mobile plate contiguous to the port's external shielding, symmetrical to the beam central axis. The rear parts of the animals were exposed in a peripheral position of the raw beam (the beam port aperture is $7.5 \mathrm{~cm}$ in radius). A protruded cone was added to the clinical BNCT beam to irradiate small animals. The neutron irradiations were performed at a reactor power of $1.004 \pm 0.004$ MW (Figure S1).

\section{Dosimetric evaluation}

Dosimetric measurements were done in mice phantoms and in vivo during irradiation. Tumor thermal flux was measured at an external position of the phantom, which was equivalent to the actual tumor position and was also in contact with the mouse. Dose in other parts of the animal body (abdomen, torso, and head) were derived from measurements in the same positions inside the phantoms replicating the positioning of the actual mice. ${ }^{14} \mathrm{~N}$ (thermal neutron dose rate) and ${ }^{10} \mathrm{~B}$ dose rates were determined from tissue thermal neutron kerma factors and the thermal flux in each position. In addition to the experimental measurements, dosimetric parameters (perturbations, cross section, etc.) were calculated using the MCNP5 calculational model of the facility.

The photon dose rate was measured using thermoluminescent dosimeters TLD-700 (Harshaw) $\left(3.2 \times 3.2 \times 0.9 \mathrm{~mm}^{3}\right)$. Estimation of the fast neutron dose was performed using the threshold inelastic reaction of the ${ }^{115} \mathrm{In}$ isotope in a pure indium massive foil $(2 \mathrm{~mm}$ thick, $20 \mathrm{~mm}$ in diameter and approximately $4.5 \mathrm{~g}$ ) which was activated by inelastic dispersion of fast neutrons with the isotope ${ }^{115} \mathrm{In}$. Thermal neutron fluxes (neutron energies below $0.5 \mathrm{eV}$ ) were measured by assessments with activation detectors whose cross-section dependence is comparable with that of nitrogen and boron (approximately $1 / \mathrm{v}$ ), such as manganese or copper.

For every group, the total absorbed dose was calculated from the addition of each absorbed dose rate component (fast neutrons, photons, and thermal neutrons) a multiplying by the irradiation time. Boron- 10 concentration was estimated to be $30 \mathrm{ppm}$ in the tumor, and $10 \mathrm{ppm}$ in normal tissues considering previous studies of BPA biodistribution measured by inductively coupled plasma 
optical emission spectroscopy (ICP-OES) (11). A 2.8\% w/w ${ }^{14} \mathrm{~N}$ concentration was assumed in every tissue, including tumor.

\section{Tumor growth measurement}

Tumor size was determined with a caliper twice a week and the volume was calculated with the following formula: $\mathrm{A}^{2} \mathrm{xB} / 2$ (where $\mathrm{A}$ is the minor diameter and $\mathrm{B}$ is the major diameter) (15). Tumor growth was evaluated between 30 and 40 -days post treatment.

\section{SIRI}

To calculate the body temperature of living subjects, standard methods that aim to limit the impact of surrounding and physiological elements that could interfere with the desired measurements must be followed. For live screening, it is essential to accomplish homeothermic conditions. Such conditions are usually achieved through control of the surrounding temperature, the infrared sources, and the undesirable stress of the observed subject (16). Two thermal cameras were employed: a Raytheon PalmIR 250 (L3 Comm. Systems, with uncooled infrared ferroelectric sensors) and a FLIR T420 (with uncooled vanadium oxide microbolometers) both comprising an array of $320 \times 240$ detectors. The detectors were sensitive to infrared radiation with wavelengths between 7 and 14 micrometres and were able to detect objects located between $0.5 \mathrm{~m}$ and infinite. By focusing the lens at $0.65 \mathrm{~cm}$ we obtained an angular field of view of $11^{\circ} \times 8^{\circ}$ and a spatial resolution of $0.39 \mathrm{~mm} /$ pixel. The total temperature resolution that includes the intrinsic sensitivity above noise and the adjustment by the analog-todigital conversion method, was around $0.3{ }^{\circ} \mathrm{C}$. The cameras also give an NTSC video signal and video acquisition capabilities that can be used for examining the temperature evolution (required in Dynamic Infrared Imaging, or DIRI, studies) or to optimize S/N Ratios through averaging video frames. Infrared cameras were frequently calibrated in temperature using reference radiation sources called black bodies. The assumed emissivity was 0.98 (a common value for normal skin) and to avoid changes in mice homeostasis, the area was maintained in a constant temperature. The NCT and both BNCT groups were examined with SIRI pre-treatment. During the measurements, mice were hand-held without sedation and infrared recordings were acquired during only a few seconds to prevent variations in the temperature of the animals due to the stress generated by the immobilization procedure.

For each mouse short recordings were acquired examining the inoculated side (right side) and left flank (normal side). On the other hand, to estimate body core temperature groin temperature was assessed with a thermocouple. All the SIRI studies were done one week before irradiation. Consequently, they could be used as an a priori non-invasive evaluation method and an indicator of boron uptake, cellular metabolism, and tissue composition (Figure S2).

\section{Hematoxylin and Eosin (HE) stains and immunobistochemistry}

At the first and $30^{\text {th }}$ day after irradiation, tumor and surrounding skin were fixed in $10 \%$ formaldehyde $(\mathrm{pH} 7.0$, PBS buffered), embedded in paraffin, cut into $10 \mu \mathrm{m}$ slices and stained with HE. Sections were deparaffinized and rehydrated through graded concentrations of ethanol. After that, the slides were transferred into a $0.05 \mathrm{M}$ Tris-based solution in $0.15 \mathrm{M} \mathrm{NaCl}$ with $0.1 \% \mathrm{v} / \mathrm{v}$ Triton-X-100, pH 7.6 (TBST). Endogenous peroxidase activity was blocked by means of a $3 \%$ hydrogen peroxide solution for $10 \mathrm{~min}$. Antigen retrieval was performed by microwave oven in citrate buffer at $\mathrm{pH}$ 6.0. Sections were then kept at room temperature for $1 \mathrm{~h}$ with an antibody: monoclonal mouse anti-rat Ki-67 (DAKO, Ely, UK). Liver and kidney samples were used as controls. For Ki-67, to obtain an optimal expression of micro vessels with the lowest value of acceptable background, the titer of primary antibody used was 1:50. Negative controls were performed replacing the primary antibodies with diluents. Following wash with PBS-Tween, sections were treated for 30 minutes at room temperature with biotinylated goat anti-mouse $\operatorname{IgG}(1: 1000$; Cell Marque) and incubated with Strepavidin-HRP (Sigma) in the same conditions.

Diaminobenzidine (DAB) was used as the chromogen and hematoxylin as the counterstain. Sections were mounted for examination. A $0.25 \mu \mathrm{m}$ per pixel resolution was used for image analysis using a ScanScope XT. The Image Pro Plus program was used to analyze the intensity of antibody labeling.

A rectangular band was scanned in a histological section, taking consecutive images, covering the central area of each histological section of approximately $300 \times 6,400 \mu \mathrm{m}$, which includes all the tumor growth. This analysis allowed evaluating the response at different cellular levels. 
Table 1 Dosimetry of mice injected with and without BPA

\begin{tabular}{lcccccr}
\hline & Boron & Thermal neutrons & Photons & Fast neutrons & Total & Total without ${ }^{10} \mathrm{~B}$ \\
\hline Tumor & 6.12 & 0.51 & 4.93 & 1.78 & 13.34 & 7.22 \\
Abdomen & 0.90 & 0.22 & 4.17 & 1.12 & 6.42 & 5.52 \\
Torso & 0.66 & 0.16 & 3.94 & 1.04 & 5.81 & 5.15 \\
Head & 0.47 & 0.12 & 3.32 & 0.49 & 4.39 & 3.92 \\
\hline
\end{tabular}

Dose rate components obtained through the calculation model in MCNP for the body regions of dosimetric interest. The total absorbed dose rates with and without boron (B) are shown (cGy/min). The uncertainties of the total dose rates in tumor are about $3 \%$ while in the non-tumor regions are about $5 \%$. Uncertainties associated with the boron compound or nitrogen content in tissues are not considered. BPA, boronophenylalanine.

Table 2 Dosimetry

\begin{tabular}{lcc}
\hline & $\begin{array}{c}\text { Irradiation time } \\
\text { (minutes) }\end{array}$ & $\begin{array}{c}\text { Total tumor } \\
\text { absorbed dose (Gy) }\end{array}$ \\
\hline NCT & $37 \pm 0.5$ & 2.67 \\
BNCT I & $37 \pm 0.5$ & 4.95 \\
BNCT II & $55 \pm 0.5$ & 6.88 \\
\hline
\end{tabular}

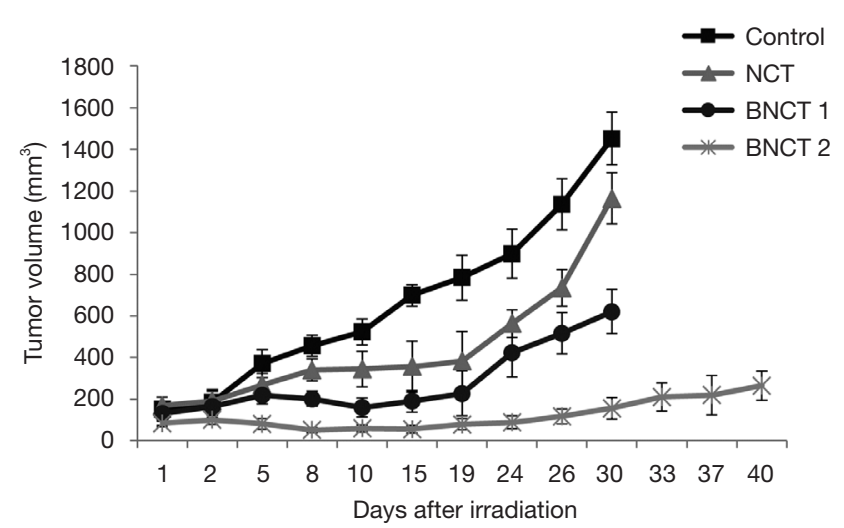

Figure 1 Absolute tumor volume $\left(\mathrm{mm}^{3}\right)$ as a function of the time post irradiation for each treatment (Control; NCT; BNCT I and BNCT II). Each point value is the mean \pm SEM of 9-12 animals of two independent experiments.

\section{Statistical analysis}

Results are shown as mean \pm SEM of 9-12 samples of two independent experiments. Temperature measurements, on the other hand, were represented by single observations. One-way ANOVA followed by Bonferroni test was used to analyze tumor growth and cell proliferation studies. Differences were considered significant when $\mathrm{P}<0.05$.

\section{Results}

\section{Irradiations at the RA-6 BNCT neutron beam}

The tumors were positioned at a radial distance of $7.5 \mathrm{~cm}$, which corresponds to a $98 \%$ of the maximum thermal neutron flux measured at the center of the beam. Tables 1 and 2 show the results of doses for the in-air thermal flux $\left(4.96 \times 10^{8} \pm 0.05 \% \mathrm{n} / \mathrm{cm}^{2} / \mathrm{s}^{\wedge}(-1)\right.$ fast neutron dose rate, ${ }^{14} \mathrm{~N}$ dose rate, photon dose rate and ${ }^{10} \mathrm{~B}$ dose rate for tumor, abdomen, torso and head of the animals. The tumor total absorbed doses were 2.67 Gy for NCT group; 4.94 and 6.88 Gy for BNCT I and BNCT II, respectively.

\section{Tumor growth}

Tumors in the animals of the Control group continued to grow resulting in an average absolute value of $1,452.23 \mathrm{~mm}^{3}$ at 30 days post treatment. At the same endpoint time, the NCT group reached an average tumor volume of $1,417.82 \mathrm{~mm}^{3}$, while both BNCT groups showed a complete growth inhibition during the first 20 days after treatment, then the tumor started to grow again reaching values of absolute tumor volume of 620.42 and $156.28 \mathrm{~mm}^{3}$, respectively at 30 days $(\mathrm{P}<0.001)$. The ratios between the tumor volumes of Control/NCT group was 1.25; Control/ BNCT I was 2.3 and Control/BNCT II was 9.29 (Figure 1).

The individual responses for BNCT I and BNCT II were analyzed 40 days after treatment, the relative tumor volume, this is the relationship between the final volume (Vf) and the initial volume $(\mathrm{Vi})$, was calculated and considered partial response to treatment (PR) when $\mathrm{Vf} / \mathrm{Vi}<1$ and $>0$. The complete response to treatment (CR) was considered when $\mathrm{Vf} / \mathrm{Vi}=0$. (Table S1). A greater tumor control effect was observed in the BNCT II group compared to the 
A

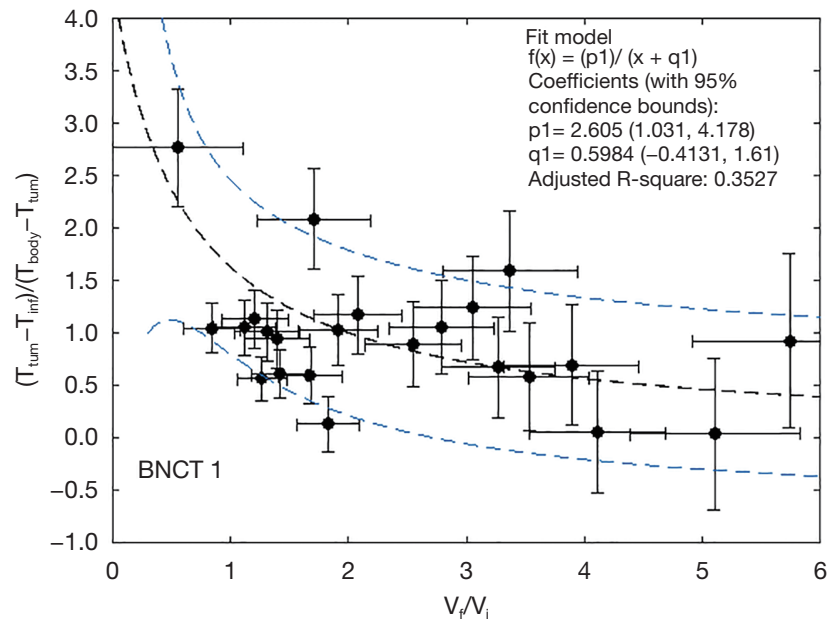

B

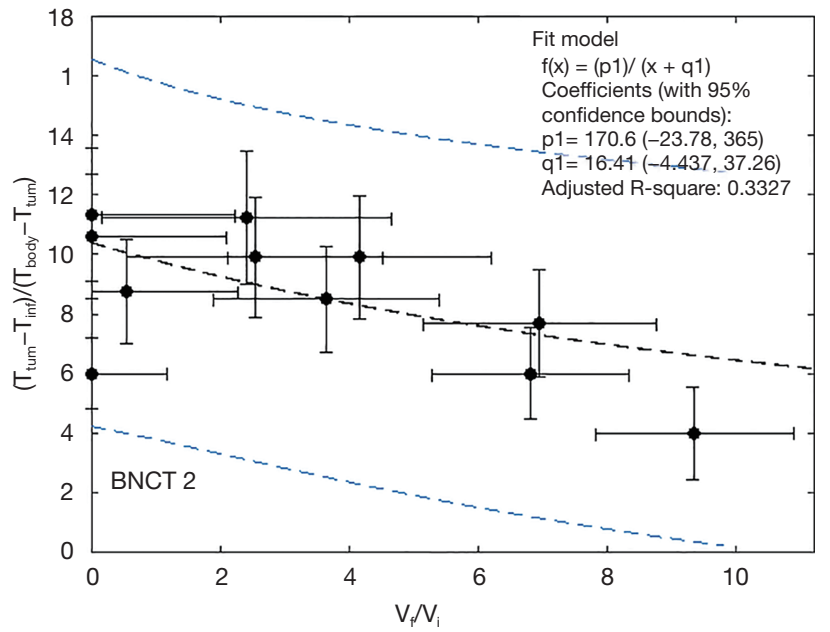

Figure 2 Ratio of temperature differences (see text) $v s$. final and initial tumor volume ratios for the BNCT I (2A) and BNCT II (2B) protocol. The temperature ratio quantity is proportional to the tumor thermal conductivity, being lower for those tumors that progressed after irradiation. Conversely, tumors with a delayed grow were those which had higher values of the thermal quantity.

BNCT I group. The partial response to the treatment was $8.7 \%$ for the BNCT I group and $9.1 \%$ for the BNCT II group. In addition, we observed a complete response to the treatment in the $27.3 \%$ of BNCT II group. Besides, in the BNCT I group the complete response was $0 \%$. This would show that an increase in the dose delivered to the tumors (around 2 Gy) could cause a considerable improvement in the tumor response to the therapy.

\section{Individual analysis post treatment}

An individualized study of the animals of BNCT I and BNCT II groups, was carried out. Pretreatment tumor temperature and its relationship with the tumor response were also analyzed.

Body temperature and tumor temperature were measured for each mouse using SIRI prior to irradiation, as a noninvasive method to correlate boron uptake by the tumor with the response to treatment. Tables S2 and S3 show the body temperature and pre-irradiation tumor temperature data and the difference between each of them and ambient temperature (Tinf) for BNCT I and BNCT II respectively. The ambient temperature for BNCT I was $20 \pm 2{ }^{\circ} \mathrm{C}$ and $28 \pm 2{ }^{\circ} \mathrm{C}$ for BNCT II. The relative error of the volume quotients was $15 \%$ and the relative error temperature quotient was $20 \%$ (although they are not shown in the table to improve the visualization). The individualized temperature data was compared with the relative final volume of each animal. Figure $2 A, B$ show the relationship between the parameters (T tum-Tinf)/ (T body-T tum) and the $\mathrm{Vf} / \mathrm{Vi}$ ratios.

The surface temperature ( $\mathrm{T}$ tum) is the result of two competing heat transfer processes: the heat produced by the mouse's body conducted through the tumor and the same amount of heat dissipated to the ambient by natural convection.

Depending on the relative strength of each process, ( $\mathrm{T}$ tum) will be closer to either the body temperature ( $\mathrm{T}$ body) or to the ambient temperature ( $\mathrm{T}$ inf). Both body and ambient temperatures are not easily controlled and highly variable, therefore the goal here is to find a dimensionless quantity that will give information about the tumor conductivity, a physical quantity which is ultimately the result of the tumor composition (viable and necrotic mass fractions).

In the following we assumed that the ambient convection, symbolized by the convective coefficient $h$, was the same throughout the different thermal studies (the same immobilization was adopted, and no air currents were applied).

Although the tumor size should be considered as well, in this first approach we assumed the same for all measurements on average.

Owing to the mathematical similarity between the 

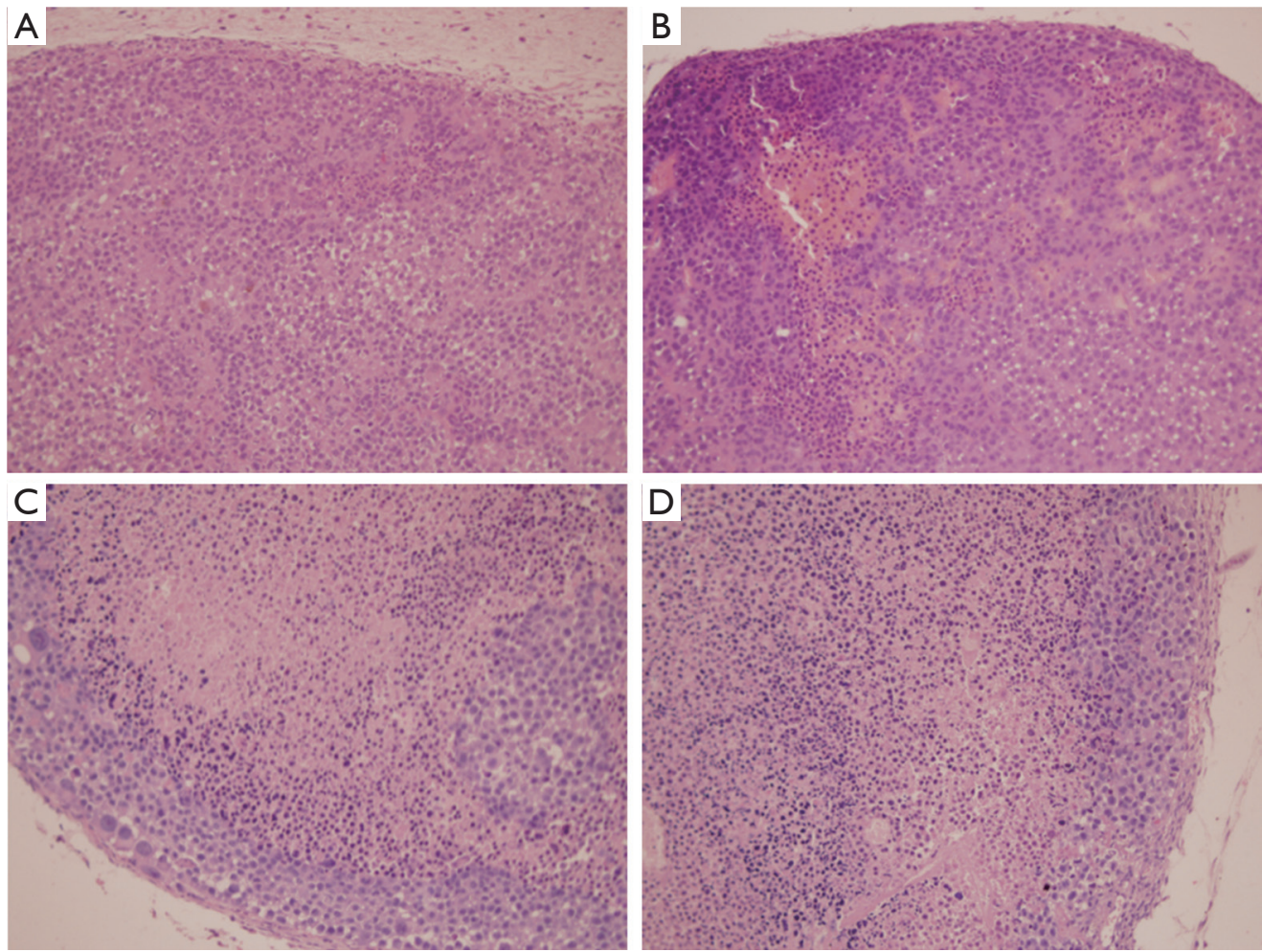

Figure 3 Comparison of viability and necrosis areas in tumors of the Control and BNCT II groups at 24 hours and 30 days after irradiation. Representative micrographs of hematoxylin and eosin stained tumor sections $(\times 10)$ are shown. At 24 hours post irradiation, the Control group presents large areas of viability with a higher number of mitotic cells (A). At the same time, the BNCT II group showed an area of necrosis with pyknotic nucleus (B). At 30 days post irradiation, the Control group presented a necrotic central area and an important viable peripheral zone, indicating tumoral growth $(\mathrm{C})$. At the same time, the BNCT II group presented a central zone of necrosis and a small viable peripheral zone indicating the beginning of tumor growth (D).

Fourier's law of heat conduction and the Ohm's law of electrical conduction, this process can be represented as an electrical circuit, where the electric current is equivalent to the heat current, the electric potentials in the different points are associated with the temperatures, and the resistances are associated with the inverse of the conductivity, and the inverse of the convective force transferring heat into the ambient (Figure S3). In the analog electrical circuit, the electrical current is the same along the circuit, then by Ohm's law of electrical conduction, (VB$\mathrm{VC}) /(\mathrm{VA}-\mathrm{VB}))=\mathrm{R} 2 / \mathrm{R} 1$. By this analogy, it can be seen that the quantity (T_tum-T_inf /(T_body-T_tum) is, among other quantities, proportional to the inverse of the tumor resistance, i.e., it is proportional to the tumor conductivity.

With this simple approach, we have just used the measurable quantities by infrared thermography, to see for the first time if tumor control and tumor temperature prior to irradiation are correlated. Although the correlation was somehow weak (BNCT I with a $\mathrm{R}^{2}$ of 0.3527 , BNCT II with a $\mathrm{R}^{2}$ of 0.3327 ), a tendency to higher values of the temperatures ratios was observed with respect to the degree of tumor control, paving the way to better describe, in terms of a more accurate model, the observed results (work in progress).

\section{Histological and immunobistochemical studies}

The tumor histology of BNCT II and Control groups at $24 \mathrm{~h}$ post treatment were compared (Figure $3 A, B$ ). Tumors with similar sizes of both groups presented large areas of viability. In the Control Group (A) no necrotic areas and presence of mitotic cells were observed; on the contrary, in the BNCT group areas of necrosis and picnotic regions were observed evidencing important tumor damage. On 

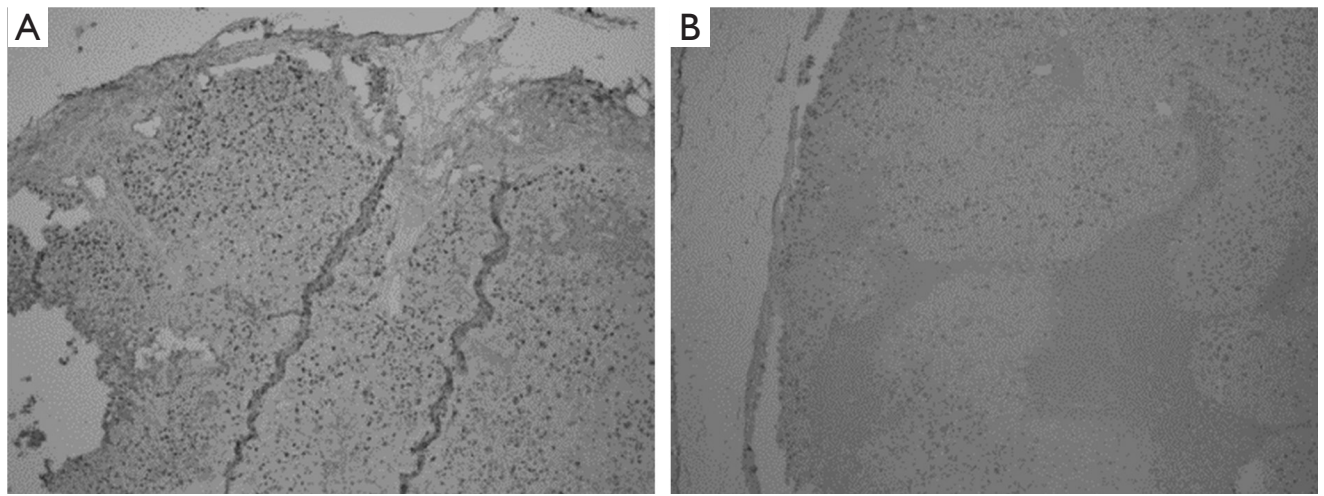

Figure 4 Tumor proliferative state 24 hours post irradiation. Representative micrographs of samples of the Control group (A) and the BNCT II group (B) at $24 \mathrm{~h}$ post irradiation, are shown. The Control group showed a more intense signal mark of the Ki-67 antibody than the BNCT II Group. The BNCT therapy could be acting on the proliferative state of the tumor at 24 h post irradiation. Magnification $\times 10$.

the other hand, the comparison between these two groups at the end of the experiment (30 days post irradiation), showed tumors significantly greater in size for the Control group respect to the BNCT II group. Large central areas of necrosis and peripheral areas of viability were observed in the Control group (Figure $3 C$ ) and a small viable peripheral zone in the Group BNCT II (Figure 3D). This demonstrated that these tumors had begun to grow again.

The immunohistochemical studies showed an important decrease of the Ki-67 antibody labeling in the BNCT II group at $24 \mathrm{~h}$ compared to Control group, indicating that the BNCT therapy acts rapidly decreasing the level of proliferation. Figure 4 shows representative photos of these groups. In Figure S4, a detail of Figure 4 is shown with a greater magnification. The BNCT therapy would be acting on the proliferative state of the tumor at 24 post irradiations.

\section{Discussion}

In the current studies, we wanted to show that there is a relationship between tumor characteristics (making special emphasis on temperature) and the response to BNCT treatment for the CM. The idea is to find out from the previous evaluation of some of these tumor characteristics, the tumor boron concentration and to be able to plan the neutron irradiation achieving better individual clinical results. Previously, we had demonstrated in experimental studies that the tumors with larger areas of viability and higher temperature had exhibited a higher BPA uptake following a rational function fit $\left(\mathrm{R}^{2}=0.7\right)(11)$.
$\mathrm{CM}$ is a tumor that originates from melanocytes and is one of the most aggressive cancers in humans. The incidence of $\mathrm{CM}$ is still increasing worldwide and is one of the most common types of cancer detected in young adults (6). CM can appear at any place on the skin surface. Even though, the location in a distinct segment of the body could be determined by the patient's sex and age. In men, $\mathrm{CM}$ grows most frequently on the back and in women it develops on the lower extremities (17). Among all skin cancers $\mathrm{CM}$ represents around $3 \%$ and constitutes the $65 \%$ of the skin cancer deaths. However, an early detection and convenient treatment of $\mathrm{CM}$ could increase the cure rate to $90 \%$ in patients with incipient melanoma (18).

Radiotherapy, along with surgery and systemic therapy, is one fundamental treatment option in cancer therapy. Patient survival could be significantly prolonged and the local control rates of tumors improved by radiotherapy. At present, different research efforts in radiation oncology are being made to overcome limitations such as using agents that targets molecules, based screening and stratification strategies, optimization of treatment planning, accuracy of dose application, and clinical implementation of other types of radiation, like protons and heavy ions (7). BNCT which produces alpha particles and ${ }^{7} \mathrm{Li}$ nuclei in the tumor tissue, has shown promising improvements compared to conventional radiotherapy. However, with both type of radiotherapies, different degrees of response have been observed for the same application of the treatment in a tumor with the same histopathologic diagnosis.

Regarding this, the Personalized Cancer Therapy is the new paradigm in medicine based on the specific tumor 
biomarkers used to help to identify potential therapy options for patients. Clinical, molecular, and anatomical imaging biomarkers are being used more frequently for treatment selection and response determination (8). Clinical investigations of BNCT for the treatment of different types of malignant melanoma have been carried out in different centers of scientific research in the world including Argentina (5). Within the framework of personalized cancer therapy, the main objective of this study was to seek the optimization of BNCT for each individual patient. Menéndez and col. have previously demonstrated in patients with CMs of extremities with the same histopathology, different degrees of response to the application of BNCT. Moreover, different skin melanoma nodules within tumor isodose surface of $15 \mathrm{RBE}$ weighted Gy, showed different grades of response after eight weeks. In those studies, a treatment plan was obtained based on the computed tomography (CT) study of the patient's extremities and the measured concentration profile of 10B in blood (19).

In previous studies, we have measured in an animal model for human melanoma different histologic, structural, and physical parameters. Our goal was to study the possible application of BNCT considering the characteristics of each patient. In that opportunity we showed that the amount of BPA incorporated by the tumor was positively correlated to the tumor temperature and with the proliferation (11). In the present work we analyzed the relationship between the individual characteristics of the tumor especially the temperature and the response to the full BNCT treatment (boron plus irradiation). The objective focused on the optimization of the delivered treatment plan by the previous estimation of boron, obtained from the individual tumor parameters.

The present studies performed with two neutrons fluences showed a dose-response dependency. The tumor growth rate was significantly diminished in the BNCT I group's animals, even though no complete remission was obtained. Contrarily, in the BNCT II group there was a complete halt of tumor growth which was evident during the 30 days of the observation period. BNCT treatment achieved a partial response with both dose schemes and a complete response for some animals $(27.3 \%)$ at the highest dose. There was an average difference of approximately 2 Gy between the two BNCT groups which represent an increase of $30 \%$ in the total absorbed dose that is reflected in the results of the BNCT treatment.

Regarding the histological and immunohistochemical studies performed, we observed that after 20 days in all the treatments (NCT or both BNCT) the tumor begins to grow after a period of complete inhibition of growth. This could be probably generated because the dose received in these animals was not enough. The proliferative status in the BNCT II group analyzed through the antibody antiKi67, was low compared to the Control group. The Ki 67 is a protein that is present during all active phases of the cell cycle (G1, S, G2 and mitosis) but it is absent in resting cells (G0), for this reason is an excellent cellular marker of cell proliferation (20). In other studies, we have demonstrated in vitro arrest in the G2 cell cycle phase by BNCT using BPA as a boron agent (21).

After the studies of SIRI, the individualized temperature data were compared with the relative final volume of each animal. In this way, it was shown the relationship between the parameters (T_tum-T_inf) /(T_body-T_tum) and the $\mathrm{VfN}$ i ratios. Considering the established analogy between the Ohm's law of electrical conduction and the heat conduction of Fourier's law, the quantity (T_tum-T_inf)/ ( $\mathrm{T}$ _body-T_tum) was, among other quantities, proportional to the tumor conductivity. This was a simple approach performed to see for the first time if tumor control and tumor temperature prior to irradiation were correlated. The correlation found was weak (BNCT I with a $\mathrm{R}^{2}$ of 0.3527 , BNCTII with a $\mathrm{R}^{2}$ of 0.3327 ), but a tendency to higher values of the temperature's ratios was observed, with respect to the degree of tumor control. Regarding this point a better description would be shown by modelling in three dimensions the complete heat transport process (work in progress).

The average body core temperature in the mice was found to be around $37^{\circ} \mathrm{C}$, distributed in a range between $35^{\circ} \mathrm{C}$ and $39{ }^{\circ} \mathrm{C}$, with a surface temperature slightly lower and more variable, depending on ambient conditions (22). It is known that several pathological processes can induce either systemic or local thermal anomalies, e.g., increased temperature due to infection, inflammation, trauma and malignancy or decreased temperature due to ischemia. Normally, in xenograft models, tumors have extremely high growth rates and therefore the vasculature is substantially different from that of normal tissue. In fact, tumor vessels are more permeable, smaller, and poorly organized. All these characteristics contribute to reduce the temperature of the tumor as the tumor grows (23). On the other hand, regarding to the material composition, soft tissues can be described as built primarily with three physical materials: water, fat, and protein. Among these materials, proteins have the lowest heat conductivity while water has the 
highest one. Because of this, heat will be conducted more efficiently in tumors with higher water content in different compartments. All heat sources, like those originated from inflammation or cellular metabolism will always cause an increase in tumor temperature (24). This same behavior was observed in this study. The smaller tumors exhibited the highest temperatures at the initial stage of the treatment, in contrast with large tumors that exhibited the greatest differences compared to body temperature.

Thermal imaging is a technique that has been employed in clinical medicine since early 1960. As the results of SIRI can be negatively influenced by different external factors, technical considerations and patient preparation must be controlled. For example, both the study room and the patient must be appropriately prepared. If our pre-clinical studies were confirmed in human clinical trials for CM, infrared thermography could provide a valuable tool device to evaluating noninvasively and in advance the potential variations in the boron uptake of the tumor. Hence, this could add to develop a personalized BNCT treatment plan. The improvement of the BNCT treatment plan delivered could allow an early escalation (e.g., increasing radiation dose or addition of other alternative therapy), de-escalation (i.e., reducing overtreatment and unnecessary toxicity in patients with good prognosis) or switch to another treatment modality.

\section{Conclusions}

Both, the histological and immunohistochemical characteristics on the one hand, and the temperature of each tumor measured by SIRI on the other, allow us to understand the type of tumoral response to BNCT, making it possible in the future to plan a personalized treatment for each patient.

\section{Acknowledgments}

We would like to thank the English Language Professor Griselda Pereyra who provided language editing assistance of the manuscript.

Funding: Our studies were supported by National Atomic Energy Commission and grants from National Agency for Scientific and Technological Promotion and National Council of Scientific and Technical Research.

\section{Footnote}

Reporting Checklist: The authors have completed the
ARRIVE reporting checklist. Available at http://dx.doi. org/10.21037/tro-20-61

Conflicts of Interest: All authors have completed the ICMJE uniform disclosure form (available at http://dx.doi. org/10.21037/tro-20-61). Dr. MAD reported grants from National Atomic Energy Commission, National Agency for Scientific and Technological Promotion and National Council of Scientific and Technical Research, during the conduct of the study. The other authors have no conflicts of interest to declare.

Ethical Statement: The authors are accountable for all aspects of the work in ensuring that questions related to the accuracy or integrity of any part of the work are appropriately investigated and resolved. The study was conducted in accordance with the Declaration of Helsinki (as revised in 2013). Experiments were performed under a project license (Protocol No3/2015 "Preclinical studies to optimize the application of BNCT for the treatments of melanoma and undifferentiated thyroid cancer") granted by the Argentine National Atomic Energy Commission Animal Care and Use Committee (CICUAL-CNEA) in compliance with the National Institute of Health (NIH USA) guide for the care and use of Laboratory animals.

Open Access Statement: This is an Open Access article distributed in accordance with the Creative Commons Attribution-NonCommercial-NoDerivs 4.0 International License (CC BY-NC-ND 4.0), which permits the noncommercial replication and distribution of the article with the strict proviso that no changes or edits are made and the original work is properly cited (including links to both the formal publication through the relevant DOI and the license). See: https://creativecommons.org/licenses/by-nc-nd/4.0/.

\section{References}

1. Barth RF, Zhang Z, Liu T. A realistic appraisal of boron neutron capture therapy as a cancer treatment modality. Cancer Commun (Lond) 2018;38:36-42.

2. Mishima Y. editor. Selective thermal neutron capture therapy of cancer cells using their specific metabolic activities-melanoma as prototype. In: Cancer neutron capture therapy. New York: Plenum Press, 1996:1-26.

3. Mishima Y, Ichihashi M, Hatta S, et al. New thermal neutron capture therapy for malignant melanoma: melanogenesis-seeking 10B molecule-melanoma cell 
interaction from in vitro to first clinical trial. Pigment Cell Res 1989;2:226-34.

4. Fukuda H, Honda C, Wadabayashi N, et al. Pharmacokinetics of 10B-p-boronophenylalanine in tumours, skin and blood of melanoma patients: a study of boron neutron capture therapy for malignant melanoma. Melanoma Res 1999;9:75-83.

5. González SJ, Bonomi MR, Santa Cruz GA, et al. First BNCT treatment of a skin melanoma in Argentina: dosimetric analysis and clinical outcome. Appl Radiat Isot 2004;61:1101-5.

6. Bandarchi B, Jabbari CA, Vedadi A, et al. Molecular biology of normal melanocytes and melanoma cells. J Clin Pathol 2013;66:644-8.

7. Orth M, Lauber K, Niyazi M, et al. Current concepts in clinical radiation oncology. Radiat Environ Biophys 2014;53:1-29.

8. Kurnit KC, Bailey AM, Zeng J, et al. "Personalized Cancer Therapy": A Publicly Available Precision Oncology Resource. Cancer Res. 2017;77:e123-6.

9. Santa Cruz GA, González SJ, Bertotti J, et al. First Application of Dynamic Infrared Imaging in Boron Neutron Capture Therapy for Cutaneous Malignant Melanoma. Med Phys 2009;36:4519-29.

10. Williams KL, Williams FJ, Handley RS. Infra-red thermometry in the diagnosis of breast disease. Lancet 1961;2:1378-81.

11. Carpano M, Perona M, Rodriguez C, et al. Experimental studies of boronophenylalanine (10BPA) biodistribution for the individually tailored application of boron neutron capture therapy (BNCT) for malignant melanoma treatment. Int J Radiat Oncol Biol Phys 2015;93: 344-52.

12. Guerra L, Mordoh J, Slavutsky I, et al. Characterization of IIB-MEL-J: A New and Highly Heterogenous Human Melanoma Cell Line. Pigment Cell Res 1989;2:504-9.

13. US Department of Health and Human Services. Guide for the care and use of laboratories animals. Public Health Service, National Institute of Health;1985. NIH Publication 86-23.

14. Coderre JA, Chanana AD, Joel, DD, et al. Biodistribution of boronophenylalanine in patients with glioblastoma multiforme: boron concentration correlates with tumor cellularity. Radiat Res 1998;149:163-70.

15. Lee YS, Bullard DE, Zalutsky MR, et al. Therapeutic efficacy of antiglioma mesenchymal extracellular matrix 131I-radiolabeled murine monoclonal antibody in a human glioma xenograft model. Cancer Res 1988;48:559-66.

16. Santa Cruz GA, González SJ, Dagrosa MA, et al. Dynamic infrared imaging for biological and medical applications in boron neutron capture therapy. In: Safai M, Brown J. editors. Thermosense: Thermal Infrared Applications XXXIII. Proceedings of SPIE, 2011:801307.

17. Lesage C, Barbe C, Le Clainche A, et al. Sex-related location of head and neck melanoma strongly argues for a major role of sun exposure in cars and photoprotection by hair. J Invest Dermatol 2013;133:1205-211.

18. Nestle FO, Kerl H. Melanoma. In: Bolognia JL, Jorizzo JL, Rapini RP, et al. editors. Melanoma in Dermatology. Toronto: Mosby, 2003:1789-815.

19. Menéndez PR, Roth BM, Pereira DM, et al. BNCT for skin melanoma in extremities: updated argentine clinical results. Appl Radiat Isot 2009;67:S50-3.

20. Rieger E, Hoffman-Wellenhof R, Soyer H et al. Comparison of proliferative activity as assessed by proliferating cell nuclear antigen (PCNA) and Ki 67 monoclonal antibodies in melanocityc skin lesions. J Cutan Pathol 1993;20:229-36.

21. Rodriguez C, Carpano M, Curotto P, et al. In vitro studies of DNA damage and repair mechanisms induced by BNCT in a poorly differentiated thyroid carcinoma cell line. Radiat Environ Biophys 2018;57:143-52.

22. Kelly G. Body temperature variability (Part 1 ): a review of the history of body temperature and its variability due to site selection, biological rhythms, fitness, and aging. Altern Med Rev 2006;11:278-93.

23. Song C, Appleyard V, Murray K, et al. Thermographic assessment of tumor growth in mouse xenografts. Int J Cancer 2007;121:1055-8.

24. Funda DP, Houstek J, Holub M, et al. Differences in thermoregulation between inmunocompetent and immunodeficient hairless mice exposed to mild cold. Folia Microbiol (Praha) 1998;43:487-9.

doi: $10.21037 /$ tro-20-61

Cite this article as: Carpano M, Santa Cruz G, Rodriguez C, Nievas S, Olivera MS, Perona M, Boggio E, Longhino J, Pisarev M, Juvenal G, Dagrosa MA. Experimental studies for the personalized application of boron neutron capture therapy to the treatment of cutaneous melanoma. Ther Radiol Oncol 2021;5:8. 


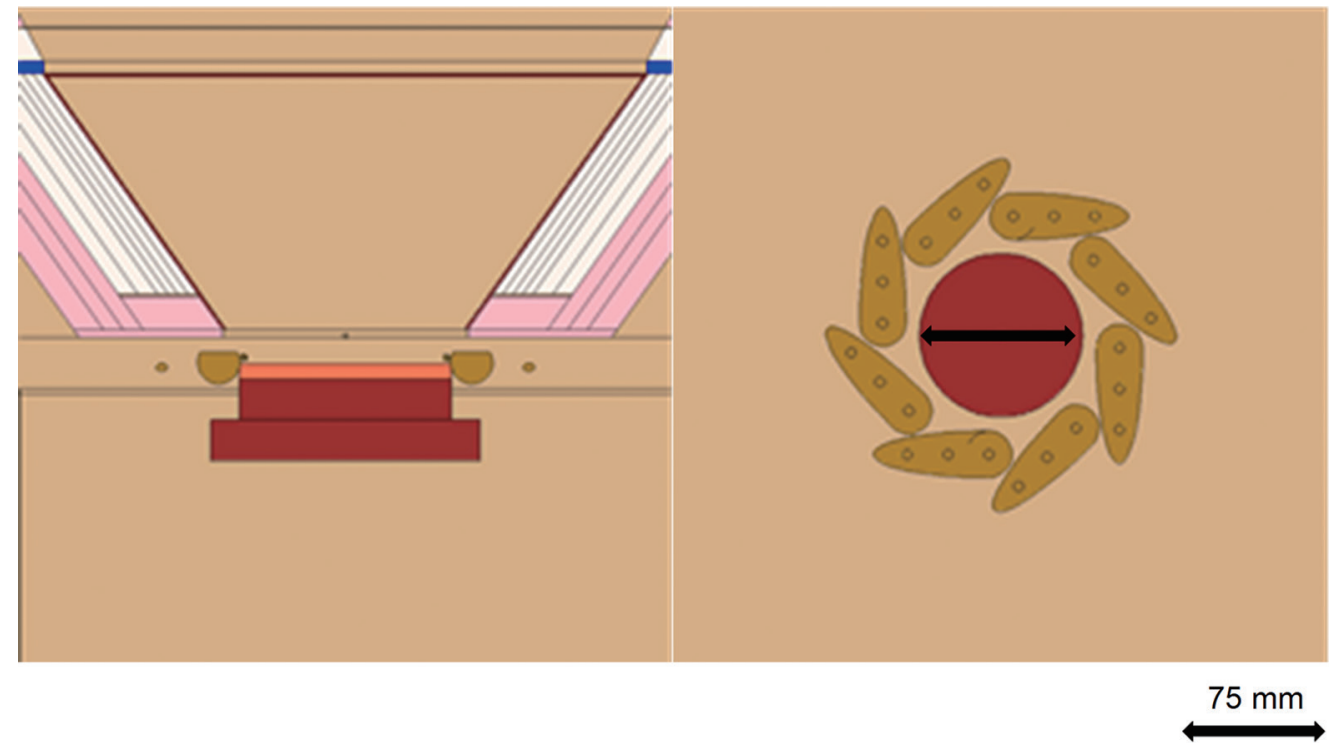

Figure S1 Experimental setup for small animal irradiations. Left: protruding cone and neutron reflector transversal to the beam axis direction. Right: small animals positioning, on the periphery of the neutron reflector, beam axis view.

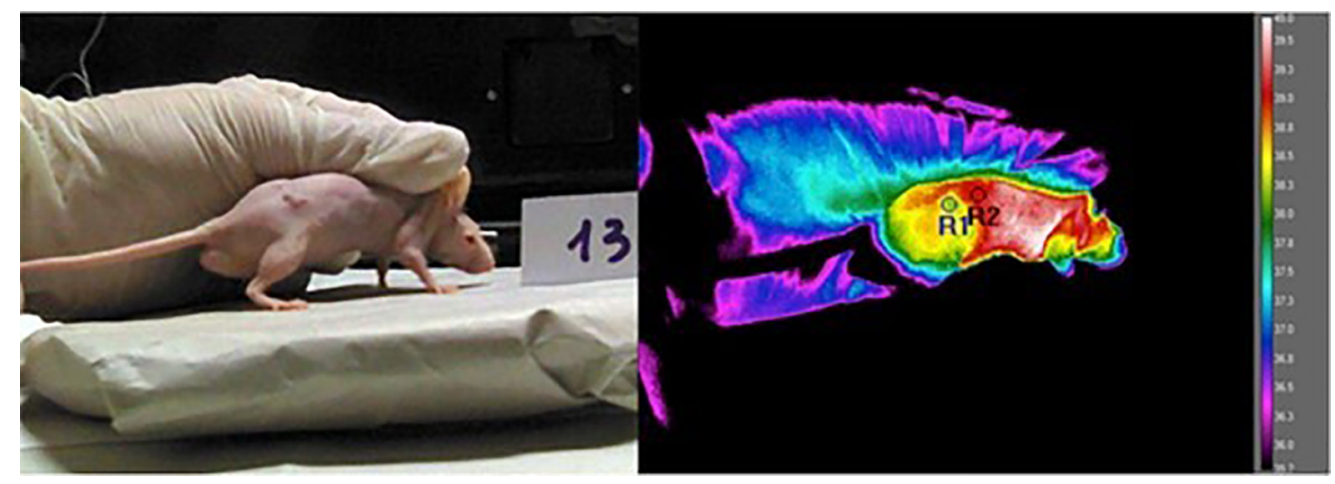

Figure S2 Visible (left) and infrared (right) images of thermography studies taken prior to BNCT treatments. R1 and R2 (infrared image) are examples of selected regions for tumor and normal tissue temperature measurements. 
Table S1 Response to the BNCT treatment

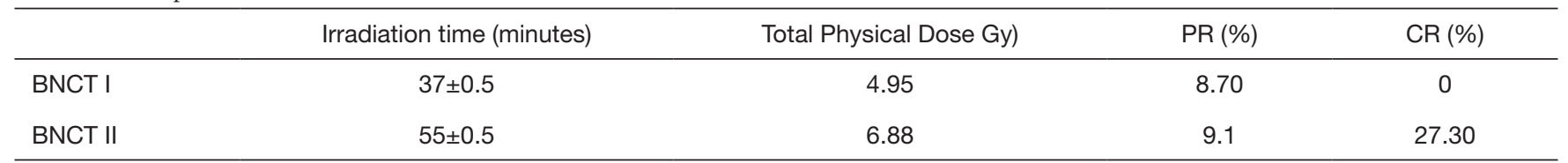

The comparison response of the BNCT I and the BNCT II groups, is shown. For the BNCT I group, the received physical dose was 4.95 Gy and for the BNCT II group it was $6.88 \mathrm{~Gy}$. PR: Vf/Vi $<1$ and $>0, \mathrm{CR}$ : Vf/Vi $=0$. PR, partial response; CR, complete response.

Table S2 BNCT I

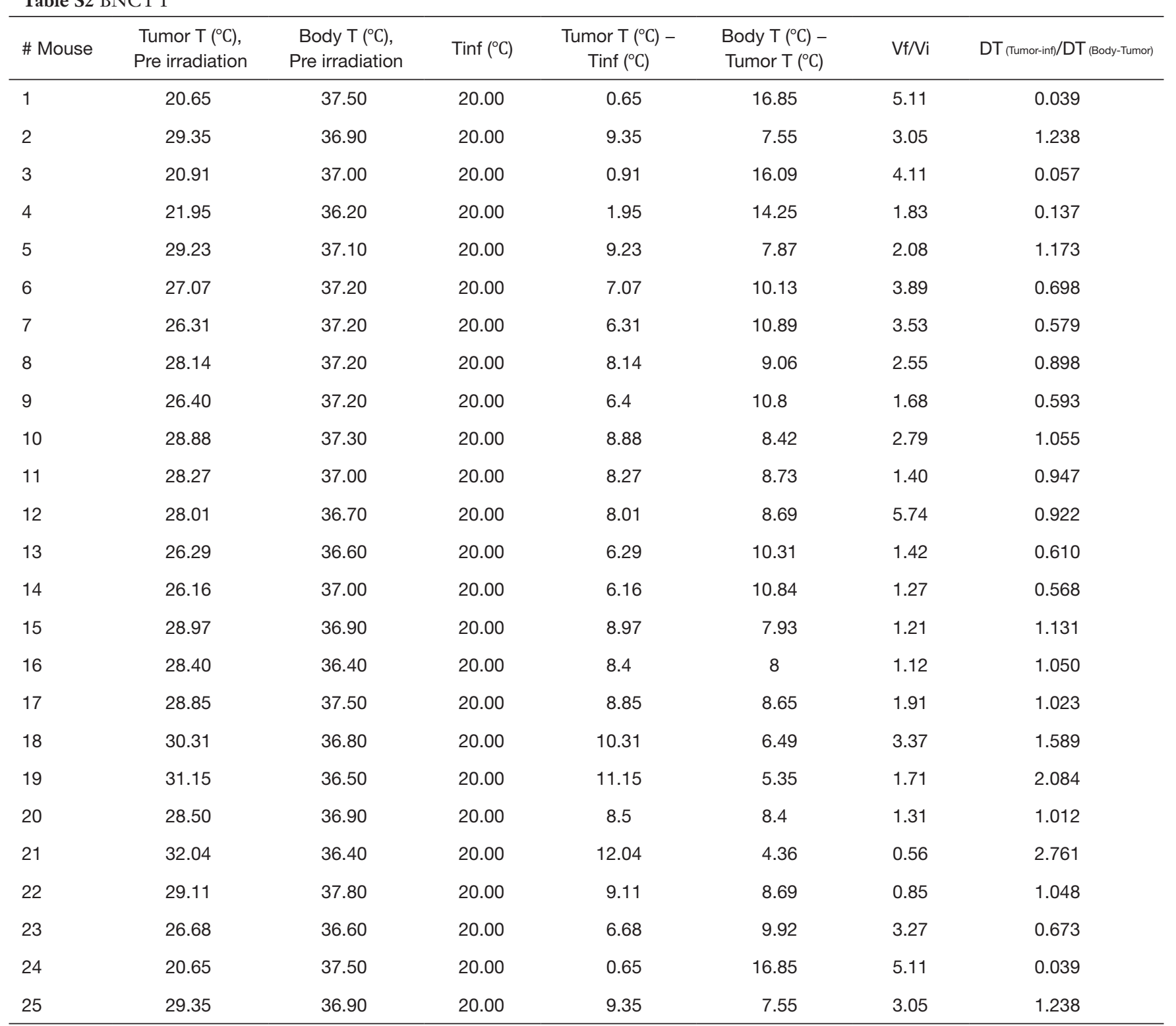

For each mouse of BNCT I group the tumor and body temperatures pre irradiation in ${ }^{\circ} \mathrm{C}$, (measured by SIRI), $\mathrm{T}$ inf and the different parameters calculated $\left[\operatorname{Tumor} \mathrm{T}\left({ }^{\circ} \mathrm{C}\right)-\operatorname{Tinf}\left({ }^{\circ} \mathrm{C}\right)\right.$; Body $\left.\mathrm{T}\left({ }^{\circ} \mathrm{C}\right)-\operatorname{Tumor} \mathrm{T}\left({ }^{\circ} \mathrm{C}\right)\right]$ and the Vf/Vi ratio for each tumor. 


\begin{tabular}{|c|c|c|c|c|c|c|c|}
\hline \# Mouse & $\begin{array}{l}\text { Tumor } \mathrm{T}\left({ }^{\circ} \mathrm{C}\right) \text {, } \\
\text { Pre irradiation }\end{array}$ & $\begin{array}{c}\text { Body } \mathrm{T}\left({ }^{\circ} \mathrm{C}\right) \text {, } \\
\text { Pre irradiation }\end{array}$ & $\operatorname{Tinf}\left({ }^{\circ} \mathrm{C}\right)$ & $\begin{array}{c}\text { Tumor } \mathrm{T}\left({ }^{\circ} \mathrm{C}\right)- \\
\operatorname{Tinf}\left({ }^{\circ} \mathrm{C}\right)\end{array}$ & $\begin{array}{l}\text { Body } \mathrm{T}\left({ }^{\circ} \mathrm{C}\right)- \\
\text { Tumor T }\left({ }^{\circ} \mathrm{C}\right)\end{array}$ & $\mathrm{Vf} / \mathrm{Vi}$ & DT (Tumor-inf)/DT (Body-Tumor) \\
\hline 1 & 37.90 & 38.90 & 28.00 & 9.90 & 1.00 & 4.17 & 9.90 \\
\hline 2 & 37.20 & 39.50 & 28.00 & 9.20 & 2.30 & 9.36 & 4.00 \\
\hline 3 & 38.10 & 39.00 & 28.00 & 10.10 & 0.90 & 2.41 & 11.22 \\
\hline 4 & 38.20 & 39.10 & 28.00 & 10.20 & 0.90 & 0.00 & 11.33 \\
\hline 5 & 37.90 & 38.90 & 28.00 & 9.90 & 1.00 & 2.54 & 9.90 \\
\hline 6 & 38.50 & 39.70 & 28.00 & 10.50 & 1.20 & 0.55 & 8.75 \\
\hline 7 & 38.20 & 39.90 & 28.00 & 10.20 & 1.70 & 6.81 & 6.00 \\
\hline 8 & 38.00 & 39.30 & 28.00 & 10.00 & 1.30 & 6.96 & 7.69 \\
\hline 9 & 38.20 & 39.40 & 28.00 & 10.20 & 1.20 & 3.65 & 8.50 \\
\hline 10 & 38.60 & 39.60 & 28.00 & 10.60 & 1.00 & 0.00 & 10.60 \\
\hline 11 & 38.20 & 39.90 & 28.00 & 10.20 & 1.70 & 0.00 & 6.00 \\
\hline
\end{tabular}

For each mouse of BNCT II group the tumor and body temperatures pre irradiation in ${ }^{\circ} \mathrm{C}$, (measured by SIRI), $\mathrm{T}$ inf and the different parameters calculated $\left[\right.$ Tumor $\mathrm{T}\left({ }^{\circ} \mathrm{C}\right)-\operatorname{Tinf}\left({ }^{\circ} \mathrm{C}\right)$; Body $\left.\mathrm{T}\left({ }^{\circ} \mathrm{C}\right)-\operatorname{Tumor} \mathrm{T}\left({ }^{\circ} \mathrm{C}\right)\right]$ and the Vf $/ \mathrm{Vi}$ ratio for each tumor.

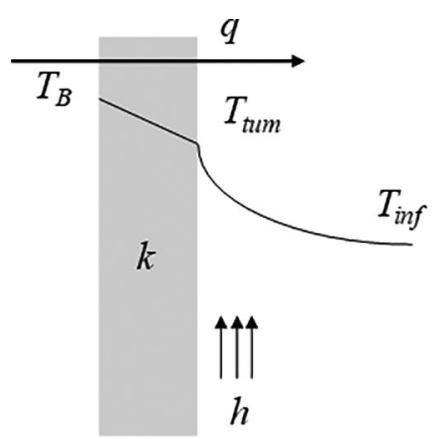

Thermal conduction

Fourier's Law

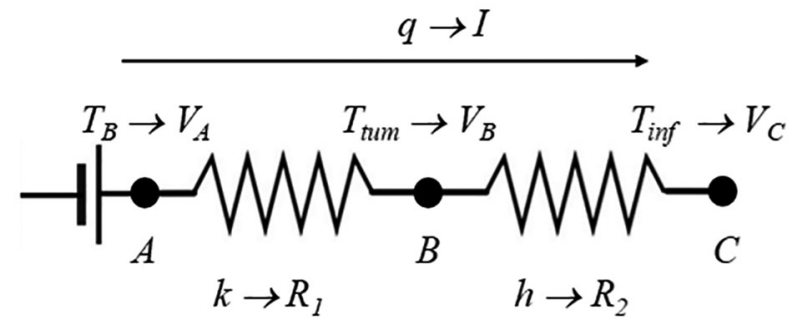

Electric conduction

Ohm's Law

Figure S3 Electrical conduction analogy based on the functional dependence of the Fourier thermal conduction law. This analogy permits obtaining a quantity proportional to physical properties only, independent of local and ambient values (assuming that natural convection is the same throughout the different measurements), suitable for comparing protocols where very different temperature values are measured. 
A

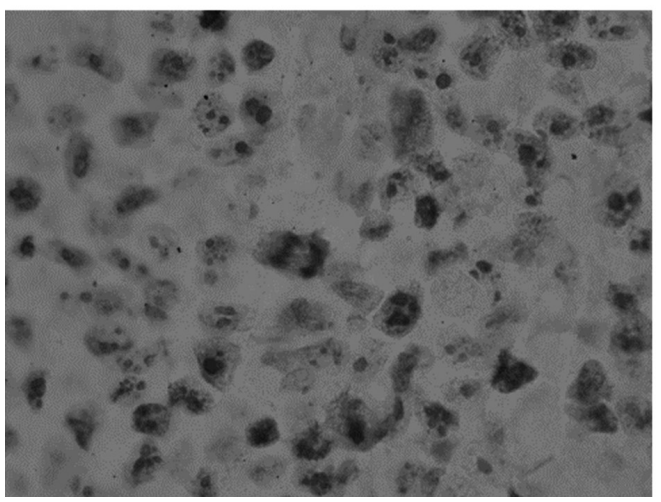

B

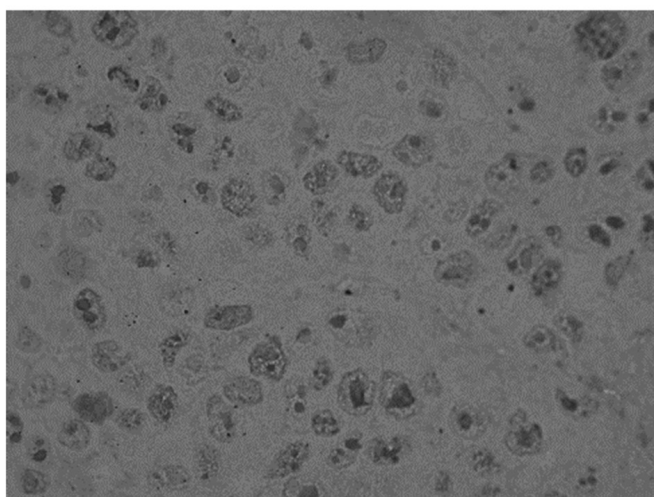

Figure S4 Tumor proliferative state at 24 hours post irradiation. Representative micrograph of samples from the Control group (A) and samples from the BNCT II group (B) 24 hours after irradiation are shown. The Control Group showed a stronger label of Ki-67 antibody staining than the BNCT II group. The BNCT therapy could be acting on the proliferative state of the tumor at 24 post irradiations. Magnification $100 \times$. 